\title{
Corrigendum: Macrophage-secreted granulin supports pancreatic cancer metastasis by inducing liver fibrosis
}

Sebastian R. Nielsen, Valeria Quaranta, Andrea Linford, Perpetua Emeagi, Carolyn Rainer, Almudena Santos, Lucy Ireland, Takao Sakai, Keiko Sakai, Yong-Sam Kim, Dannielle Engle, Fiona Campbell, Daniel Palmer, Jeong Heon Ko, David A. Tuveson, Emilio Hirsch, Ainhoa Mielgo and Michael C. Schmid

Nature Cell Biology 18, 549-560 (2016); published online 18 April 2016; corrected after print 20 May 2016

In the version of this Article originally published, in the last sentence of the 'Acknowledgements' section, 'Pancreas Biomedical Research Unit' should have read 'National Institute for Health Research Biomedical Research Unit funding scheme through a NIHR Pancreas BRU'. This has been corrected in all online versions of the Article.

\section{Erratum: Oncogenic mTOR signalling recruits myeloid- derived suppressor cells to promote tumour initiation}

Thomas Welte, Ik Sun Kim, Lin Tian, Xia Gao, HaiWang, June Li, Xue B. Holdman, Jason I. Herschkowitz, Adam Pond, Guorui Xie, Sarah Kurley, Tuan Nguyen, Lan Liao, Lacey E. Dobrolecki, Lan Pang, Qianxing Mo, Dean P. Edwards, Shixia Huang, Li Xin, Jianming Xu, Yi Li, Michael T. Lewis, Tian Wang, Thomas F. Westbrook, Jeffrey M. Rosen and Xiang H.-F. Zhang

Nature Cell Biology 18, 632-644 (2016); published online 16 May 2016; corrected after print 20 May 2016

In the version of this Article originally published, in the fourth affiliation, 'Los Angeles' should have read 'Louisiana'. This has been corrected in all online versions of the Article. 